\title{
Considerações Sobre a Avaliação Psicológica no $\operatorname{Brasil}_{*}($
}

\section{Considerations about Psychological Assessment in Brazil}

Consideraciones acerca de Evaluación Psicológica en Brasil
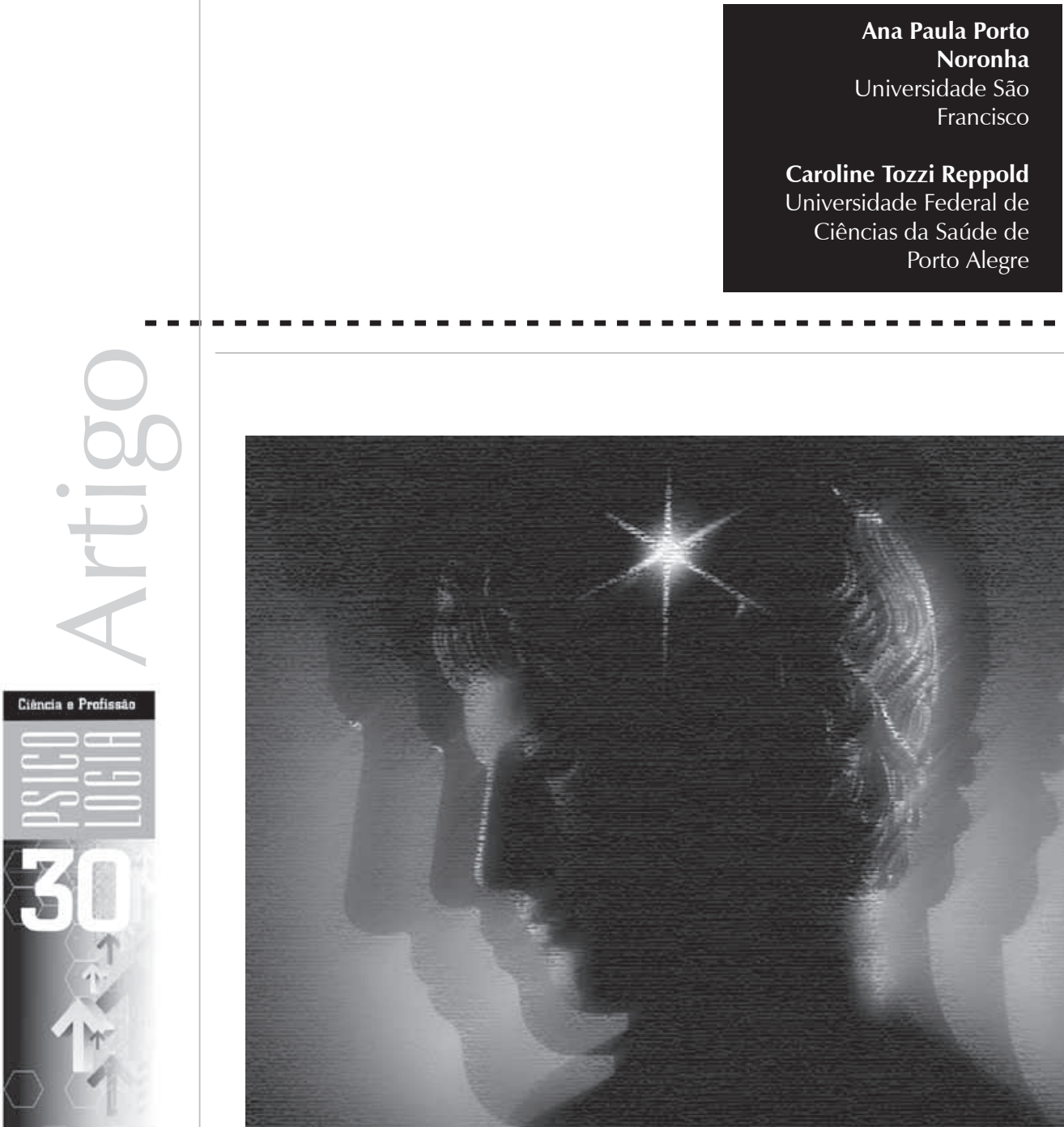

(*) As autoras

são membros da diretoria do Instituto Brasileiro de Avaliação Psicológica (IBAP), sendo a primeira, a presidente passada, e a segunda, a futura presidente. 
Resumo: O presente artigo foi elaborado em virtude da comemoração dos 30 anos da revista Psicologia: Ciência e Profissão, já que no primeiro número, havia um trabalho sobre avaliação psicológica, a mesma temática aqui trabalhada. O objetivo a que se propôs foi resgatar o desenvolvimento da área de avaliação psicológica no Brasil bem como refletir sobre seu momento atual e sobre as necessidades futuras para o aprimoramento das práticas avaliativas, da formação dos psicólogos e da área de conhecimento. A título de finalização, as autoras fazem menção aos preceitos de que progresso científico-tecnológico está relacionado ao desenvolvimento do País, de modo que países mais desenvolvidos tendem a ter melhores condições de avaliação. Especialmente no que respeita a avaliação, espera-se que, em um futuro próximo, os psicólogos a realizem com mais propriedade, que os instrumentos psicológicos tenham as qualidades psicométricas necessárias e que a sociedade a reconheça como uma prática científica.

Palavras-chave: Avaliação psicológica. História da Psicologia. Testes psicológicos. Formação do psicólogo.

Abstract: This paper was written due to the 30th year celebration of the journal Psicologia: Ciência e Profissão, since there was a work on psychological assessment in the first issue, the same theme approached in this paper, that paper aimed at retrieving the development of psychological assessment field as well as pondering about its current moment and its future needs for the improvement of assessing practices, psychologists' education and the field of expertise. As conclusion, the authors refer to the precept that the scientific and technological progress is related to the development of the country, so that the most developed countries tend to have better assessment conditions. Especially regarding assessment, it is expected that psychologists carry it out more properly in a near future, that psychological instruments have the necessary psychometric qualities, and that society recognizes it as a scientific practice.

Keywords: Psychological assessment. History of psychology. Psychological tests. Psychologist education.

Resumen: El presente artículo ha sido elaborado en virtud de la celebración de los 30 años de la Revista Psicología Ciencia y Profesión, ya que en la primera edición había un trabajo acerca de evaluación psicológica, misma temática desarrollada aquí. El objetivo propuesto ha sido el de rescatar el desarrollo del área de Evaluación Psicológica en Brasil, así como hacer reflexión acerca de su momento actual y acerca de las necesidades futuras para el perfeccionamiento de las prácticas evaluativas, de la formación de los psicólogos y del área de conocimiento. A título de finalización, las autoras hacen mención a los preceptos de que el progreso científico tecnológico se halla relacionado al desarrollo del país, de forma que países más desarrollados son más susceptibles de tener mejores condiciones de evaluación. Especialmente en lo que tañe a la evaluación, se espera que en un futuro cercano los psicólogos la realicen con más propiedad, que los instrumentos psicológicos tengan las cualidades psicométricas necesarias y que la sociedad la reconozca como una práctica científica.

Palabras clave: Evaluación Psicológica. Historia de la Psicología. Testes Psicológicos. Formacion del psicólogo.

${ }^{1}$ Os três grupos citados são os seguintes: Pesquisa em avaliação psicológica, coordenado pela Profa. Dra. Acácia Aparecida Angeli dos Santos; Métodos projetivos nos contextos da avaliação psicológica, coordenado pela Profa. Dra. Anna Elisa Villemor-Amaral; Avaliação psicológica e neuropsicológica de crianças e adolescentes, coordenado pelo Prof. Dr. Claudio Simon Hutz.
A revista Psicologia: Ciência e Profissão completa 30 anos, e a presente edição comemorativa arrola textos que abordam temáticas publicadas no primeiro número, o que inclui a avaliação psicológica. Para a área, isso é motivo de orgulho, uma vez que, dentre assuntos diversos e históricos da Psicologia, cabe a ela uma referência. Nesse ensejo, o presente artigo tem como objetivo a reflexão sobre a avaliação psicológica de ontem, de hoje e do que se deseja para o amanhã.

\section{Contextualização da avaliação psicológica}

O cenário da área tem se modificado, ao longo dos últimos anos, mais especialmente, a partir da segunda metade da década de 1990 (Reppold \& Serafini, no prelo; Wechsler \& Guzzo, 1999). A título de justificativa da asserção, cita-se o XIII Simpósio de Pesquisa e Intercâmbio Científico em Psicologia, promovido pela Associação Nacional de Pesquisa e Pós-Graduação em Psicologia, no presente ano, no qual três grupos de trabalho ${ }^{1}$ (GTs) de avaliação estiveram presentes. Divididos por temáticas de interesse comum aos pesquisadores, esses grupos ilustram a complexidade da área de avaliação psicológica que, cada vez mais, busca abrir seu escopo de interesse e articular estudos relacionados à avaliação psicométrica, à avaliação projetiva e à avaliação neuropsicológica. 
${ }^{2}$ Mesa-redonda coordenada por André Jacquemin, que contou com a participação de Claudio Hutz, Cícero Vaz e Latife Yazigi.

${ }^{3}$ Trabalho apresentado por Latife Yazigi.

${ }^{4}$ Integravam esse GT os seguintes pesquisadores:

Cícero Vaz, Claudio Hutz, Denise Bandeira, Elisa Yoshida, Elisabeth do Nascimento, Irai Cristina Boccato Alves, Luis Pasquali, Marcelo Tavares, Solange Wecshler e Vera Figueiredo (ANPEPP, 1998).
Nesse evento, estiveram presentes aproximadamente 70 pesquisadores, de quase 30 instituições de ensino superior, de 10 Estados brasileiros e de 4 Regiões do País. $\mathrm{O}$ intuito do encontro foi problematizar e estabelecer parcerias para o desenvolvimento de projetos de pesquisa (Associação Nacional de Pesquisa e Pós-Graduação em Psicologia [ANPEPP], 2010). Os números absolutos, se olhados com descuido, podem trazer ao leitor uma interpretação de pequenez. No entanto, uma retomada histórica indica que, desde sua primeira edição, o Simpósio conta com a participação de expressivos pesquisadores da área e com estudos que buscam contextualizar o escopo da avaliação psicológica bem como articulá-la a outras áreas de conhecimento. No primeiro simpósio em que a área esteve representada, foram apresentados os seguintes trabalhos: Interação, cultura e personalidade: contribuição das técnicas projetivas $^{2}$ e A prova de Rorschach, a epilepsia temporal e a especialização hemisférica ${ }^{3}$.

Desde 1998, quando o Simpósio, em sua 7ạ edição, assumiu como forma de organização a constituição de grupos de trabalho, o grupo Pesquisa em avaliação psicológica integrou as reuniões da ANPEPP. Coordenado em Gramado, RS pelo prof. Luís Pasquali e pela profa Dra. Solange Wechsler ${ }^{4}$ (Weschler, 1998), o grupo foi posteriormente coordenado pelos professores Claudio Simon Hutz, Ricardo Primi e Ana Paula Noronha, e, em 2008, redistribuído em razão de seu superdimensionamento e do crescimento expressivo de laboratórios e de linhas de pesquisa relacionadas à avaliação psicológica (ANPEPP, 2010).

Produções importantes foram oriundas das discussões promovidas pelos pesquisadores da área. Exemplos disso são os documentos considerados guidelines. Dentre eles, a tradução autorizada das Diretrizes para Uso de Testes da International Test Commission, realizada pelo Instituto Brasileiro de Avaliação
Psicológica - IBAP, em colaboração com o Laboratório de Avaliação e Medidas Psicológicas (LAMP) da PUC-Campinas (http:// www.ibapnet.org.br/docs/DiretrizesITC. PDF) e o manifesto elaborado em 2002 pelos membros do então GT Pesquisa em Avaliação Psicológica (http://www.ibapnet. org.br/avalpsi_manifesto.html). Nessa ocasião, pesquisadores se reuniram e elaboraram um documento intitulado Em defesa da Avaliação Psicológica, no qual sugeriam conteúdos que deveriam fazer parte dos currículos de formação do psicólogo brasileiro na área de avaliação psicológica (AP). Teoria da medida e psicometria, avaliação da inteligência e da personalidade e prática de planejamento, execução e redação de resultados foram destacados pelos autores como conteúdos programáticos imprescindíveis para disciplinas da área (Noronha et al., 2002). Cite-se também a participação de membros do grupo que, por intermédio do IBAP, colaboraram com o Conselho Federal de Psicologia (CFP) no que se refere à implantação, em 2003, do Sistema de Avaliação dos Testes Psicológicos (SATEPSI). Essa iniciativa resultou na elaboração de critérios de avaliação da qualidade dos testes psicológicos e em uma lista de testes que apresentam evidências científicas para utilização profissional. Nessa situação, uma das intervenções propostas pelos pesquisadores foi a organização de dúvidas levantadas na lista de discussão virtual AvalPsi sobre o SATEPSI, as quais foram enviadas ao CFP, que as respondeu por meio de um documento para consulta divulgado nos sites do CFP e do IBAP (ANPEPP, 2004). Essas referências ilustram que, além de projetos de pesquisas, a articulação dos pesquisadores tem propiciado a efetivação de manifestações e de intervenções públicas em prol da avaliação.

Aliado a isso, há outros números interessantes que podem realçar o estado da arte e indicar o crescimento da área em questão. Um deles refere-se à revista Avaliação Psicológica. Esse 
periódico temático foi criado em 2002, sob a editoração do prof. Claudio Hutz, inicialmente com uma participação modesta de publicações. Com o passar dos anos, o aumento de manuscritos submetidos foi tão intenso que houve necessidade de alteração na periodicidade da revista, que passou a ter três números por ano. Desde sua fundação até o momento, foram publicados 19 números, nos quais aproximadamente 150 artigos inéditos sobre AP foram apresentados (http://scielo.bvs-psi.org.br/www.ibapnet. org.br), revelando o crescente interesse de pesquisadores pela avaliação.

Há décadas a avaliação também se organiza como associação científica e elemento ativo nos movimentos políticos da Psicologia brasileira. Nesse sentido, estão o Instituto Brasileiro de Avaliação Psicológica - IBAP (www.ibapnet.org.br) e a Associação Brasileira de Rorschach e outros métodos projetivos ASBRo (http://www.asbro.org.br), fundados, respectivamente, em 1997 e 1993 com o intuito de promover o desenvolvimento da área e de representar a avaliação psicológica em órgãos e instituições de interesse do psicólogo. Além da organização de eventos científicos, da formação de grupos de pesquisa e das frequentes discussões sobre a formação profissional, essas instituições têm colaborado com importantes decisões que envolvem a Psicologia no País. Suas participações no Fórum das Entidades Nacionais da Psicologia Brasileira - FENPB, ao lado de outras 21 entidades, é exemplo disso.

Em acréscimo, pode-se mencionar o convite recebido pelo Instituto Brasileiro de Avaliação Psicológica para participar do VII Congresso Nacional de Psicologia. O evento foi promovido pelo Conselho Federal de Psicologia - CFP em 2010, e reuniu os diretores eleitos pelos Conselhos Regionais. Além desses, cinco entidades foram convidadas, dentre elas o IBAP. Esse evento é entendido como instância máxima de deliberação da Psicologia brasileira, e seu intuito foi discutir as políticas que deverão ser adotadas pelo CFP nos próximos três anos (http://cnp.pol.org.br). Pela primeira vez, a avaliação psicológica esteve representada.

Outro dado que reflete o incremento da área é a quantidade de testes psicológicos comercializados na atualidade. Para ilustrar esse fato, faz-se necessário comentar que, em uma consulta ao Buros Institute of Mental Measurement, em 2002, (http:// www.unl.edu/buros/), foi possivel identificar aproximadamente 2000 títulos disponíveis naquele universo. Na mesma época, no Brasil, pari passu com as Resoluções no 25/2001 e no 02/2003, do Conselho Federal de Psicologia (CFP, 2010), promulgadas com o intuito de regulamentar o uso e a comercialização dos testes psicológicos, aproximados 30 instrumentos faziam parte da primeira relação do Sistema de Avaliação dos Testes Psicológicos (SATEPSI). Hoje, os psicólogos contam com 114 instrumentos, ou seja, ao longo de sete anos, o que se construiu foi muito superior ao produzido em décadas passadas, o que representa um progresso para a AP e traz repercussões para diversas outras áreas da Psicologia. Nos campos da Psicologia do desenvolvimento e da Psicologia clínica, por exemplo, dispor de instrumentos válidos auxilia na identificação precoce de padrões comportamentais disfuncionais que podem evoluir para transtornos mentais na vida adulta bem como possibilita a avaliação da eficácia de novas intervenções propostas, ou da extensão de danos neurofuncionais associados a psicopatologias específicas.

Ainda no campo clínico, citado aqui a título ilustrativo, a preocupação metodológica relacionada à padronização e à busca de evidências de validade dos instrumentos utilizados em pesquisas é fomentada, no campo da saúde mental, desde a década de 60. A proliferação do uso desses instrumentos se deve, em grande parte, 
${ }^{5}$ Membros da Comissão Consultiva em Avaliação Psicológica em 2002: Álvaro José Lelé, Audrey S. de Souza, José Carlos Tourinho e Silva, Regina Sônia G. F. do Nascimento e Ricardo Primi; 2005: Blanca Werlang, Carlos Henrique

S. S. Nunes, Maria Cristina Ferreira, Regina Sônia G. F. do Nascimento e Ricardo Primi; 2008: Blanca Werlang, Maria Abigail de Souza, Maria Cristina Ferreira, Marcelo Tavares e Ricardo Primi; 2009: Blanca Werlang, Carlos Henrique

S. S. Nunes, Maria Cristina Ferreira, Marcelo Tavares e Ricardo Primi; 2010: Anna Elisa Villemor-Amaral, Blanca Werlang, Carlos Henrique S.

S. Nunes, Caroline

T. Reppold, Maria Cristina Ferreira, Marcelo Tavares e Ricardo Primi. a dois fenômenos: a introdução de um sistema classificatório multiaxial no campo da psiquiatria a partir do DSM-III (American Psychiatric Association, 1980) e o avanço da psicofarmacologia. A proposta do DSMIII contribuiu com o desenvolvimento da psicometria por disponibilizar critérios diagnósticos operacionais para avaliação dos transtornos mentais, já a psicofarmacologia, por ressaltar a necessidade de homogeneização das amostras em pesquisas envolvendo medicamentos psicotrópicos. Assim, técnicas validadas passaram a ser consideradas uma estratégia para eliminar o aspecto subjetivo que as entrevistas traziam às avaliações. Entretanto, muitos testes criados nesse período não tiveram, até a virada do século, novos estudos de validade ou de adaptação dos instrumentos para uso nacional.

Assim, a comissão consultiva do SATEPSI desenvolveu uma árdua tarefa relacionada à avaliação dos testes disponíveis no País. A comissão que compõe esse Sistema é formada por psicólogos Doutores, especialistas em avaliação psicológica em áreas distintas. $\mathrm{O}$ primeiro grupo que a compôs teve a tarefa de estruturar, com base em parâmetros internacionais (AERA, APA \& NCME, 1999), os requisitos mínimos a partir dos quais os instrumentos deveriam ser analisados. Desde a sua criação, em 2002, a comissão consultiva já teve cinco composições diferentes ${ }^{5}$, tendo ocupado sua função profissionais que contribuem com publicações científicas, pesquisas e que exercem a docência na área de AP.

Quanto às publicações científicas, Suehiro e Rueda (2007) fizeram uma análise das produções da revista Avaliação Psicológica desde seu primeiro número, em 2002, até 2007. Os autores analisaram 100 artigos, com base em critérios como tipo de estudo, região geográfica de origem do estudo e referências empregadas, dentre outros. Os dados obtidos revelam o aumento das publicações a partir de 2005, sendo o relato de pesquisa a forma mais frequente de submissão. É importante ressaltar que, ao longo do período analisado, a revista passou da classificação CAPES/ANPEPP de $C$ Nacional para A Nacional, e atualmente inicia um processo de internacionalização, o que tende favorecer a divulgação da revista para outros países, além de poder ser enriquecida com a contribuição de outros autores.

Recentemente, observa-se também, em outros veículos, uma série de publicações que apresentam revisões sobre o estado da arte da avaliação psicológica (Joly, Martins, Abreu, Souza, \& Cozza, 2005; Joly, Silva, Souza, \& Nunes, 2007; Reppold \& Hutz, 2008; Villemor-Amaral, 2008) e neuropsicológica (Capovilla, 2007; Seabra, Dias, \& Trevisan, 2010). Somam-se a eles estudos de revisão sobre o ensino e a formação em AP (Joly, Berberian, Andrade, \& Teixeira, 2010; Noronha, Carvalho, Miguel, Sousa, \& Santos, 2010) e a aplicação da avaliação psicológica em diferentes contextos (Gurgel, Plentz, Joly, \& Reppold, 2010; Oliveira, Santos, Noronha, Boruchovitch, Cunha, Bardagi, \& Domingues, 2007; Silva \& Alchieri, 2008).

Dentre os resultados que se destacam, estão os estudos de Joly et al. (2007, 2010). Os autores destacam que, em painéis apresentados em congressos brasileiros de avaliação psicológica, assim como nas pesquisas de mestrado e doutorado na área, predominam estudos que buscam investigar os parâmetros psicométricos de testes, sobretudo de testes relacionados à avaliação de personalidade e inteligência e de uso no âmbito clínico ou escolar.

Não se pode, com isso, ingenuamente concluir que a avaliação psicológica brasileira se encontra em nível de excelência e pode ser equiparada à das grandes potências internacionais, com sua centena de testes. Sopesando, pode-se afirmar que ela está 
renascendo, o que, por certo, causa estranheza. O presente artigo é motivado pela publicação de um trabalho de Luis Pasquali (1979) em um dos periódicos que mais representam a psicologia brasileira, há 30 anos. Àquela época, o autor já se preocupava com a validação de instrumentos de medida, no caso específico, um instrumento sobre avaliação parental. Pode-se, a partir disso, afirmar que a avaliação já teve, também em décadas passadas, seus dias de glória.

O uso do Teste de Inteligência de Binet em 1907, em Minas Gerais, e a respectiva adaptação desse instrumento, em 1924, na Bahia, a publicação, por Medeiros e Albuquerque, em 1924, do primeiro livro brasileiro sobre testes psicológicos, a inauguração de centros de pesquisa como o Instituto de Seleção e Orientação Profissional (ISOP) e o Serviço Nacional de Aprendizagem Industrial (SENAI), na primeira metade do século $X X$, revelam que as experiências iniciais com avaliação psicológica no País ocorreram em um momento histórico no qual a Psicologia em si ainda era incipiente. Como explicar, por exemplo, que, em 1927, Pierón lecionou a disciplina Psicologia Experimental e Psicometria, em São Paulo, quando a Psicologia ainda não era uma profissão reconhecida oficialmente? (Noronha \& Alchieri, 2002). Isso posto, pode-se admitir que a área surgiu precocemente, vinculada às demandas sociais da época, e criou expectativas de que o seu desenvolvimento seria promissor.

A precocidade da área pode ser confirmada pela pesquisa de Noronha, Primi e Alchieri (2004). Nesse estudo, os autores analisaram as principais informações de 146 testes psicológicos comercializados no Brasil, considerando as informações dispostas nos seus manuais. Os resultados indicaram que, do total de instrumentos, 52,1\% foram elaborados até 1969, quando a profissão de psicólogo ainda estava nascendo no País. O estudo computou as produções publicadas até 2002, e, à época, os autores reforçaram a importância de uma ação mais enfática no que diz respeito ao incremento da qualidade dos testes psicológicos; adicionalmente, chamaram a atenção para o estabelecimento de formação profissional mais adequada na área em questão. Nick (1988) já havia informado que, entre 1930 e 1960, a construção de testes foi mais acelerada no Brasil, embora não tenha havido grandes preocupações com os parâmetros psicométricos, possivelmente em razão do pouco desenvolvimento dos recursos tecnológicos disponíveis na época.

A trajetória da avaliação psicológica brasileira foi também retratada por Pasquali e Alchieri (2001). Os autores estabeleceram cinco períodos pelos quais a ciência psicológica passou desde 1836, considerando o status da área de avaliação em cada época. Segundo os autores, a área apresentou um entusiasmo inicial exagerado relativo ao funcionalismo americano pós-guerra, que se manteve até o período que coincide com a regulamentação dos cursos de Psicologia. Assim, o declínio da AP ocorreu entre as décadas de 1960 a 1970, quando a escassa oferta de formação especializada trouxe como consequência um quadro de desqualificação docente e baixa qualidade do ensino. Somado ao declínio da produção na área, os testes passaram a ser duramente criticados e a ter um uso minimizado nos anos 80. Outros fatores elencados para a involução da área foram o radicalismo ideológico que acompanhou a popularização dos enfoques humanista e dialético na compreensão do ser humano e a baixa qualidade do material editorial produzido. Em decorrência, o ostracismo instalou-se, e o desenvolvimento da área ficou estagnado por quase três décadas.

Tal fato, embora não esteja sendo revelado ineditamente, ainda traz para os psicólogos que defendem a avaliação psicológica sequelas desastrosas (Noronha et al., 2010; 
Um estudo realizado por

Frizzo, em 2004,

sobre as infrações éticas mais comumente cometidas

pelos psicólogos inscritos no Conselho Regional de Psicologia 08 no período de 1994 a 2003 revelou que as denunciadas com maior frequência $(46,15 \%)$ se referem ao exercício da avaliação psicológica
Pasquali \& Alchieri, 2001, dentre outros). O abandono da avaliação impediu seu avanço, de modo que poucas pesquisas foram realizadas e um mínimo de instrumentos brasileiros foi construído. Até a criação dos primeiros centros e laboratórios de pesquisa em AP vinculados a programas de pós-graduação (CPP/USPRP-1978, LabPam/ UnB- 1987, Litep/IPUSP-1988, Lamp/ PUCCAMP-1994, LM/UFRGS-1995, Lepap/ PUCMG- 1997), ainda não havia esforços e massa crítica suficientes para oferecer uma formação apropriada, e o docente da área não recebia a devida atenção de seus pares. Uma das consequências desse quadro foi o grande número de processos éticos relacionados à área, além do notável (pré) conceito de alunos, docentes e psicólogos em relação a ela (Alchieri \& Bandeira, 2002; Hutz \& Bandeira, 2003; Noronha et al., 2002).

No tocante à formação, parece claro que a falta de excelência está diretamente relacionada à incompetência profissional. Adicionalmente, após a publicação das diretrizes curriculares, diversas instituições reduziram a carga horária de disciplinas da área de avaliação psicológica (Reppold \& Serafini, no prelo), o que acarretou um treinamento superficial do uso de testes e de outros recursos, e, especialmente, uma visão fragmentada do processo avaliativo. O pouco conteúdo abordado com o futuro psicólogo nos cursos de graduação refere-se à administração e à avaliação de determinadas técnicas, e muitas vezes é permeado de um automatismo acrítico. Dessa forma, em sua maioria, os psicólogos brasileiros não têm sido formados para pesquisar ou construir materiais. Contudo, como já dito, esse não é um panorama novo, uma vez que a formação em avaliação sofreu críticas contundentes no final do século. Na década seguinte, lamentavelmente pouco se avançou em resposta às ponderações feitas por vários autores (Hutz \& Bandeira, 2003; Noronha, 2002; Villemor-Amaral, 2008).
Recentemente, Noronha et al. (2010), com base em documentos internacionais, recomendaram tópicos que deveriam ser considerados quando da formação em avaliação psicológica; conteúdo, infraestrutura e métodos de ensino foram apontados pelos autores como elementos importantes no processo de aprendizagem. Outros tópicos atuais relacionados à AP que poderiam ser incluídos nos programas das disciplinas referem-se aos avanços na área, dentre eles, a avaliação informatizada ou a validade clínica dos instrumentos psicológicos. Em acréscimo, acredita-se que as situações ilustrativas que levem os alunos a refletir sobre como agir diante de situações que envolvam aspectos éticos relacionados à avaliação psicológica é uma estratégia importante a ser considerada.

Um estudo realizado por Frizzo, em 2004, sobre as infrações éticas mais comumente cometidas pelos psicólogos inscritos no Conselho Regional de Psicologia 08 no período de 1994 a 2003 revelou que as denunciadas com maior frequência $(46,15 \%)$ se referem ao exercício da avaliação psicológica. Os motivos alegados nas denúncias envolvem principalmente as falhas quanto ao uso dos testes aplicados e à elaboração dos relatórios psicológicos.

Esses dados mais uma vez evidenciam que a formação profissional é inegavelmente um elemento nevrálgico da Psicologia. Em que pesem estas considerações, não parece que seja algo específico da avaliação psicológica, uma vez que a preparação do psicólogo prescinde de uma revisão global. Em alguma medida, essa compreensão isenta a avaliação da responsabilidade total pelas atuações profissionais inconsistentes e convida a Psicologia a refletir sobre o que se deseja do psicólogo brasileiro no presente século.

\section{A avaliação psicológica necessária}

O que se espera da avaliação como ciência e prática profissional? A resposta não é difícil, e 
espera-se que ela seja abrangente o bastante para incluir os vários olhares.

A avaliação psicológica é, por lei, prática exclusiva do psicólogo, o que, em alguma medida, Ihe concede uma reserva nesse segmento. Para o presente século, esperase que seja imputada ao profissional a competência necessária para a realização de ações com excelência, de modo que o garantido por lei seja acrescido de competência e segurança nas suas práticas avaliativas. Sustenta-se, com isso, que a avaliação seja realizada apenas por profissionais que tenham competência para tal, e não por qualquer psicólogo, já que a eles é dado o direito de avaliar.

Para tanto, a formação brasileira precisa ser repensada. A reserva de mercado somente deve ser garantida se os psicólogos, pela sua formação, tiverem condições de compreender a complexidade de um processo avaliativo e dispuserem de recursos e conhecimentos que Ihes possibilitem ponderar em que circunstâncias uma avaliação deve ser realizada, quais os melhores instrumentos a utilizar em cada caso e como interpretar, de forma contextualizada, os dados obtidos por meio da avaliação. Para tanto, é necessário que essa habilidade, prevista nas diretrizes curriculares, seja de fato desenvolvida durante o período de graduação e atualizada por meio de formação continuada. Somente assim os psicólogos poderão sair das universidades em condição de realizar processos avaliativos adequados e de fornecer diagnósticos e prognósticos seguros (leia-se: baseados em evidências empíricas e normas atualizadas) em diferentes campos de atuação. Com isso, os processos éticos referentes às atividades de avaliação deverão diminuir, até cessar. Adicionalmente, se as formações forem mais valorizadas ao longo do curso, é possível que mais psicólogos se interessem pela área, minimizando o pré-conceito e aumentando a quantidade de profissionais e recursos na área.
Sob essa mesma perspectiva, qual seja, a da formação, os psicólogos precisam incorporar, em suas práticas profissionais, a atualização continuada. Os anos de formação são necessários, mas não são suficientes para fomentar com propriedade todas as suas ações. Constantemente novos campos de atuação profissional têm se constituído, e o psicólogo tem sido a chamado a contribuir, de forma interdisciplinar, com práticas de avaliação. Contudo, ainda se faz presente, entre alguns psicólogos, a concepção equivocada de que os anos de graduação lhes permitem ter acesso aos domínios quase infinitos da ciência psicológica. É preciso rever esses dogmas; é preciso que o psicólogo brasileiro se torne um consumidor mais efetivo e regular da literatura que se produz no País ou fora dele. Em contrapartida, é preciso que os órgãos representativos de classe discutam de forma mais fundamentada a criação do título de Especialista em avaliação psicológica, bandeira defendida pelos IBAPs e por outras associações da área de AP desde o passado.

Também é desejável que os psicólogos compreendam que o fato de a avaliação ser uma prática desse profissional, dentre as tantas outras por ele realizadas, não implica sua realização por todos os formados. Assim, como ocorre nas demais áreas de atuação do psicólogo, alguns optam por umas em detrimento de outras, supostamente levando em consideração suas competências profissionais. Conjectura-se que, embora ele não esteja vinculado a determinado contexto, deve a este o mesmo respeito que tem em relação às suas preferências. Tem-se a impressão de que, no que se refere à avaliação psicológica, tem faltado respeito por parte de certos colegas.

Por fim, almeja-se o reconhecimento social da prática avaliativa. De uma vez por todas, a avaliação psicológica precisa ser compreendida como competência imprescindível ao psicólogo - como é disposto 
nas diretrizes curriculares que regem a formação profissional - e como área que requer formação específica e atualizada, o que justifica o título de Especialista. Avaliar é necessário em diferentes contextos de atuação, pois, além de fornecer informações preciosas dos aspectos psicológicos dos processos avaliados, contribui para uma atuação mais refinada e precisa. Para que esse reconhecimento se efetive, muitas instâncias precisam se unir.

A esse respeito, as palavras de Almeida (1999) são oportunas. O autor afirma que o progresso científico-tecnológico está relacionado ao desenvolvimento do país, de modo que aqueles mais desenvolvidos, com mais recursos socioeconômicos, têm mais possibilidade de investimento em pesquisas.
Órgãos de classe, associações científicas, instituições de ensino, pesquisadores e psicólogos são os autores nesse processo. Esses são os desafios a serem vencidos no presente século. Uma vez que o Conselho Federal de Psicologia já decidiu que o próximo ano temático será referente à avaliação psicológica, espera-se que os temas apontados neste artigo sejam amplamente discutidos e que os argumentos apresentados contribuam para a implementação de práticas voltadas para a qualificação do ensino, para a formação continuada e para o avanço da produção científica. Um dos fóruns já elencados para essa discussão é o $V$ Congresso Brasileiro de Avaliação Psicológica, a ser realizado pelo IBAP, em Bento Gonçalves, RS, entre os dias 01으 e 04 de junho de 2011.

\section{Ana Paula Porto Noronha}

Doutora em Psicologia, docente do Programa de Pós-Graduação Stricto Sensu, Universidade São Francisco, São Paulo - SP - Brasil

E-mail: ana.noronha@saofrancisco.edu.br

\section{Caroline Tozzi Reppold}

Doutora em Psicologia, docente da Universidade Federal de Ciências da Saúde de Porto Alegre, bolsista Produtividade Do CNPQ. Rio Grande do Sul - RS - Brasil

E-mail: reppold@ufcspa.edu.br

*Endereço para envio de correspondência:

Rua Alexandre Rodrigues Barbosa, 45, Centro, Itatiba, São Paulo - SP - Brasil CEP 13253-231 
Referências
Alchieri, J. C., \& Bandeira, D. R. (2002). Ensino da avaliação psicológica no Brasil. In R. Primi (Org.), Temas em Avaliação Psicológica (pp. 35-39). São Paulo: Casa do Psicólogo.

Almeida, L. S. (1999). Avaliação Psicológica: exigências e desenvolvimento nos seus métodos. In S. M. Wechsler \& R. S. L. Guzzo (Orgs.), Avaliação Psicológica perspectiva internacional (pp. 41-55). São Paulo: Casa do Psicólogo.

American Educational Research Association, American Psychological Association, \& National Council on Measurement in Education. (1999). Standards for Educational and Psychologicl Testing. New York: American Educational Research Association.

Associação Nacional de Pesquisa e Pós-Graduação em Psicologia. (2010). Grupos de Trabalho. Recuperado em julho de 2010, de www.anpepp.org.br

Capovilla, A. G. S. (2007). Contribuições da neuropsicologia cognitiva e da avaliação neuropsicológica à compreensão do funcionamento cognitivo humano. Cadernos de Psicopedagogia (UNISA), 11, 1-24.

Conselho Federal de Psicologia. Sistema de Avaliação dos Testes Psicológicos - SATEPSI. Recuperado em agosto de 2010, de Disponível em www.pol.org.br

Frizzo, N. P. (2004). Infrações éticas, formação e exercício profissional em psicologia. Dissertação de Mestrado, Programa de Pós-Graduação em Psicologia, Centro de Filosofia e Ciências Humanas, Universidade Federal de Santa Catarina, Florianópolis, SC.

Gurgel, L. G., Plentz, R., Joly, M. C. R. A., \& Reppold, C. T. (2010). Instrumentos de avaliação da compreensão de linguagem oral em crianças e adolescentes: uma revisão sistemática da literatura. Revista Neuropsicologia Latinoamericana, 2, 1-10.

Hutz, C. S., \& Bandeira, D. R. (2003). Avaliação psicológica no Brasil: situação atual e desafios para o futuro. In $\mathrm{O}$. $\mathrm{H}$. Yamamoto \& V. V. Gouveia (Orgs.), Construindo a psicologia brasileira: desafios para a ciência e prática psicológica (pp. 261-277). São Paulo: Casa do Psicólogo.

Joly, M. C. R. A., Berberian, A., Andrade, R. G., \& Teixeira, T. C. (2010). Análise de teses e dissertações em avaliação psicológica disponíveis na BVS-Psi Brasil. Psicologia: Ciência e Profissão, 30(1), 174-187.

Joly, M. C. R. A., Martins, R. X., Abreu, M. C. , Souza, P. R. R., \& Cozza, H. F. P. (2005). Análise da produção científica em avaliação psicológica informatizada. Avaliação Psicológica, 3(2), 121-130.

Joly, M. C. R. A.,Silva, M. C. R., Souza, M. S., \& Nunes, M. F. O.(2007). Análise da produção científica em painéis dos Congressos Brasileiros de Avaliação Psicológica. Avaliação Psicológica, 6, 239-252.

Nick, E. (1988). Vivências relativas ao trabalho em avaliação psicológica: dificuldades, limites e perspectivas para o Brasil. Anais do XVIII. Reunião Anual de Psicologia. SBPRP.

Noronha, A. P. P. (2002). Os problemas mais graves e mais frequentes no uso dos testes psicológicos. Psicologia: Reflexão e Crítica, 15(1), 135-142.

Noronha, A. P. P., \& Alchieri, J. C. (2002). Reflexões sobre os instrumentos de avaliação psicológica. In R. Primi (Org.), Temas em avaliação psicológica (pp. 19-36). São Paulo: Casa do Psicólogo.
Noronha, A. P. P., Carvalho, L. F., Miguel, F. K., Souza, M., \& Santos, A. A. (2010). Sobre o ensino de avaliação psicológica. Avaliação Psicológica, 9(1), 139-146.

Noronha, A. P. P., Primi, R., \& Alchieri, J. C. (2004). Parâmetros psicométricos: uma análise de testes psicológicos comercializados no Brasil. Psicologia: Ciência e Profissão, 24(4), 88-99.

Noronha, A. P. P., Ziviani, C., Hutz, C. S., Bandeira, D. R., Custódio, I. C. B. A., Alchieri, J. C., Borges, L. O., Pasquali, L., Primi, R., \& Domingues, S. F. (2002). Em defesa da avaliação psicológica. Avaliação Psicológica, 1(1), 173-174.

Oliveira, K. L., Santos, A. A. A. , Noronha, A. P. P., Boruchovitch, E., Cunha, C., Bardagi, M. P., \& Domingues, S. (2007). Produção científica em avaliação psicológica no contexto educacional. Psicologia Escolar e Educacional, 11, 239-252.

Pasquali, L. (1979). Concepção de pais. Um instrumento fatorial. Psicologia: Ciência e Profissão, 1(1), 137-214.

Pasquali, L., \& Alchieri, J. C. (2001). Os testes psicológicos no Brasil. In L. Pasquali (Ed.), Técnicas de Exame Psicológico TEP Manual (pp. 195-221). São Paulo: Casa do Psicólogo.

Reppold, C. T., \& Hutz, C. (2008). Investigação psicodiagnóstica de adolescentes: encaminhamentos, queixas e instrumentos utilizados em clínicas-escolas. Avaliação Psicológica, 7, 85-91.

Sbardelini, E. T. B. (1991). Os mitos que envolvem os testes psicológicos. Documenta CRP-08, 1(1), 53-57.

Seabra, A. G., Dias, N. M., \& Trevisan, B. T. (2010). Avaliação neuropsicológica das funções executivas na infância e adolescência. In C. S. Hutz (Org.), Avanços em avaliação psicológica e neuropsicológica de crianças e adolescentes, 1, (pp. 256-276). São Paulo: Casa do Psicólogo.

Silva, F. H. V. C., \& Alchieri, J. C. (2008). Revisão das pesquisas brasileiras em avaliação psicológica de habilidades e inteligência de condutores. Estudos de Psicologia, 13(1), 57-64.

Suehiro, A. C. B., \& Rueda, F. J. M. (2009). Revista Avaliação Psicológica: um estudo da produção científica de 2002 a 2007. Avaliação Psicológica, 8(1), 131-139.

Villemor-Amaral, A. E. (2008). A validade téorica em avaliação psicológica. Psicologia: Ciência e Profissão, 28, 98-109.

Wechsler, S. M. (1999). Guia de procedimentos éticos para a avaliação psicológica. In S. M. Wechsler \& R. S. L. Guzzo (Orgs.), Avaliação psicológica: perspectiva internacional (pp. 133-141). São Paulo: Casa do Psicólogo. 